\title{
LA OMISIÓN TEÓRICA EN LA INTERPRETACIÓN DE LOS RESULTADOS DE LAS INVESTIGACIONES CIENTÍFICAS
}

\section{THE THEORETICAL OMISSION IN THE INTERPRETATION OF THE RESULTS OF SCIENTIFIC RESEARCH}

\author{
Alí Antonio Morales Valera ${ }^{1}$
}

Recibido: 2019-01-02 / Revisado: 2019-03-15 / Aceptado: 2019-04-01 / Publicado: 2019-07-01

Forma sugerida de citar: Morales-Varela, A. A. (2019). La omisión teórica en la interpretación de los resultados de las investigaciones científicas. Retos de la Ciencia, 3(7), pp. 1-10. https://doi.orq/10.53877/rc.3.7.20190701.01

\section{RESUMEN}

El presente ensayo, persigue mostrar la forma como las teorías son omitidas en la interpretación de los resultados de algunas investigaciones científicas, sean de artículos científicos, trabajos de grados o tesis. Se observó la importancia teórica con independencia del enfoque de la investigación y se revisaron los casos de omisión por propia voluntad, así como los que ocurren por desconocimiento o poco conocimiento metodológico y científico. Para evitar que ocurra este inconveniente, se mencionó como sugerencia incrementar el espacio que se le da a la interpretación de resultados en los textos de metodología, así como la mayor atención a este aspecto en las asignaturas de metodología enseñadas en las instituciones de educación superior.

Palabras clave: teoría, marco teórico, investigación, metodología, interpretación de resultados.

\section{ABSTRACT}

The present essay finds to show the way in which theories are omitted in the interpretation of results of some scientific investigations, be for papers, degree works or thesis. Theoretical importance was observed independently of the research approach and cases of omission were reviewed of their own accord, as well as those that occur due to ignorance or little methodological and scientific knowledge. To avoid this inconvenience, it was mentioned as a suggestion to increase the space given to the interpretation of results in the methodology texts, as well as the greater attention to this aspect in the methodology subjects taught in higher education institutions.

Keywords: theory, theoretical framework, research, methodology, interpretation of results.

\footnotetext{
${ }^{1}$ Magíster en Tecnología Educativa. Docente agregado con dedicación exclusiva en la Universidad Nacional Experimental Politécnica de las Fuerzas Armadas. Venezuela. E-mail: amskell8@gmail.com
} 


\section{INTRODUCCIÓN}

Dentro del ámbito científico y avanzando más específicamente a la esfera educativa, los resultados obtenidos de las investigaciones, tienen un rol preponderante dentro de las mismas; de hecho, todo el andamiaje metodológico que suele usarse para desarrollarlas, cumple la función de ejecutar pasos rigurosos, confiables y verificables, que puedan asegurar que tales resultados son el producto de una labor científica estricta.

La metodología debe reflejar la estructura lógica y el rigor científico del proceso de investigación, desde la elección de un enfoque metodológico específico (preguntas con hipótesis fundamentadas correspondientes, diseños de la muestra o experimentales), hasta la forma como se van a analizar, interpretar y presentar los resultados del proyecto. (Gómez, 2006, p. 66)

Así, ya sea en la elaboración de un trabajo de grado (pregrado, especialización - maestría) o una tesis doctoral dentro del espacio educativo (todas estas consideradas investigaciones científicas en el presente contexto), o en la publicación de artículos en revistas científicas; toda investigación debe reflejar dentro de su estructura, lo que se obtuvo de la realización de esa labor y para ello se vale de la metodología. Esta última, en el contexto universitario, sigue un aspecto más normativo (Cazau, 2011, p. 114), es decir que se enseña para que los estudiantes entiendan de forma fundamental, cómo realizar una investigación; es decir, se enseña el método de una forma teórica, que luego debe ser puesto en práctica con la elaboración de alguno de los trabajos previamente mencionados, según el nivel educativo.

Precisamente, en la aplicación del método de forma práctica, surgen algunos inconvenientes que se repiten y suelen ser comunes, los cuales están asociados a los resultados de la investigación, esto no es problema menor si se recuerda que "En la práctica, la ciencia que transmite mejor sus resultados es la más útil, por ello es prioritario comunicar los resultados" (Cáceres, 2014, p. 7). Más allá de lo reflejado en esa parte de la investigación, la cual es abierta a las reflexiones del lector, es conveniente que el autor del trabajo que es quien lo conoce, presente su propia interpretación de los mismos, pues allí se observa si estos cumplieron con las expectativas teóricas iniciales en caso que existiesen, si sirvieron para generar nuevo conocimiento o refutar uno ya validado.

Lo anterior, sirve como marco de presentación para revisar una problemática observada en el ámbito de la investigación educativa y que luego de una revisión exhaustiva, se pudo identificar también en muchas publicaciones científicas en general. Se va tomar como punto de partida, la presentación de trabajos de grado para optar al título de Magister de un programa postgrado de Tecnología Educativa de la Universidad Nacional Experimental Politécnica de la Fuerza Armada (UNEFA) en Venezuela.

En el mismo orden de ideas, en el contexto mencionado previamente, el investigador ha fungido en repetidas ocasiones como jurado de este tipo de trabajo, observando en la práctica común de estos, que los resultados son presentados de forma "divorciada" de las teorías o marcos teóricos empleados, dándole a estos últimos un papel de accesorio dentro del trabajo y no el que debe cumplir a la hora de verificar o refutar hipótesis, presentar nuevos conocimientos, explicar anomalías o plantear investigaciones dentro de una teoría existente o línea de investigación asociada; es decir, que no se toman en cuenta a la hora de interpretar los resultados. 
En virtud de lo planteado, hacer una revisión de la situación que se presenta representa un trabajo epistemológico, recordando que esta disciplina (la epistemología) "analiza los supuestos filosóficos de las ciencias, su objeto de estudio, los valores implicados en la creación del conocimiento, la estructura lógica de sus teorías, los métodos empleados en la investigación y en la explicación o interpretación de sus resultados". (Batthyány y Cabrera, 2011, p. 11) por lo que, dentro de la presente revisión, se realizará un enfoque de este tipo, que permita entender lo que ocurre y como se extiende el problema fuera del contexto local, situación que afecta al conocimiento científico, por qué los métodos utilizados se emplean mal, son mal interpretados o simplemente ignorados.

De lo anterior, queda explícito el criterio epistemológico del presente opúsculo, sin embargo, existen otros argumentos por los que se justifica su realización, tales como el aspecto metodológico que se puede separar del anterior "La epistemología y la metodología son dos áreas del conocimiento que suelen tratarse conjuntamente; pero, en otras ocasiones, suele presentárselas por separado como si fueran dominios diferentes" (Cazau, 2011, p. 109), en este caso particular ocurre de esta forma, ya que se está revisando la metodología empleada en trabajos de grado y artículos científicos en general desde afuera, verificando que se estén empleando criterios con respecto al uso de las teorías en la interpretación de los resultados que validen este conocimiento, puesto que la investigación científica es un proceso que considera un producto (objeto), basado en teorías y hechos. (Samaja, 2004, p. 49)

Igualmente, aparecen argumentos académicos, ya que entender la función que cumplen las teorías dentro de las investigaciones, mejoraría los productos obtenidos, pues se quiere que estos trabajos posibiliten la generación de nuevos productos, por lo que su realización de forma rigurosa, favorecerá al punto de partida de otros dentro de la misma línea de investigación o alguna similar. Lo anterior, permitiría ubicar los resultados de las investigaciones obtenidas, dentro de alguna de las teorías de partida (o refutar algún conocimiento) disminuyendo la posibilidad de que exista un conocimiento aislado (sin relación teórica), lo cual hace que este pierda su sentido y utilidad.

\section{DESARROLLO}

En primer lugar, dentro del conocimiento que se está revisando, hay que hacer explícito que los resultados junto con la metodología, son elementos fundamentales de la investigación. En trabajos de grado o tesis, existe siempre al menos un capítulo dedicado a la presentación e interpretación de los mismos y en los artículos científicos, al menos en el sistema tradicional de presentación de estos, conocido como sistema IMRYD (introducción, método, resultados y discusión), hay incluso dos epígrafes donde se trabaja con este aspecto.

Conviene destacar, que ya la sola presentación en sí misma de los resultados muestra una falta de homogeneidad o universalidad "Los gestores de la actividad de ciencia y técnica y los evaluadores de sus resultados en diversos órganos científicos no poseen un criterio uniforme sobre la presentación de los resultados" (Travieso, 2017, p. 612) y si se revisa la interpretación que se hace de los mismos (cuando se hace) la situación tiende a empeorar.

En relación con lo anterior, se podría establecer que toda investigación, cualquiera sea su forma final de presentación, incluso si es publicada o no (caso de algunas tesis o trabajos de grado), debería incluir una interpretación de los 
resultados obtenidos, lo cual no se cumple todo el tiempo, pues a veces los investigadores, sobre todo en algunos artículos científicos, dejan intencionalmente las interpretaciones para que sean hechas por el lector, restándole a este la oportunidad de debatir con la idea del autor. Otras veces, ocurre que se hacen interpretaciones meramente estadísticas de los resultados alcanzados, discutiendo porcentajes y especulando acerca del motivo de determinadas tendencias y correlaciones, sin contrastar hipótesis iniciales (si las hubiese) contra la teoría o en el caso de elaboración de productos tecnológicos, cómo responden los resultados a los requerimientos iniciales obtenidos a partir de las teorías.

Lo anterior viene a decir que, el papel de la teoría dentro de las investigaciones, debería ser clave a la hora de entender los resultados obtenidos; sin embargo, sobre todo en las ciencias sociales, no ocurre así todo el tiempo "los posicionamientos ante la función de la teoría en las Ciencias Sociales en general y en la investigación en particular son muy variados. Van desde hacerla totalmente central hasta prácticamente ignorarla", (Dendaluze, 1997, p. 83) esto lleva a dividir el problema del uso de las mismas en la interpretación de los resultados en dos vertientes, una (la que se acaba de mencionar) que se hace de manera intencionada cuestionando al menos su importancia y otra (de donde se observó la problemática) que es omitida por desconocimiento ya sea metodológico o científico. Ambas vertientes se desarrollan a continuación.

Asimismo, para revisar el caso de la omisión intencionada del uso de la teoría en los resultados obtenidos (el cual abarca en algunos casos toda la investigación), hay que volver al sempiterno debate de enfoques, el cualitativo y el cuantitativo, que la ciencia en general no termina de superar. En el aspecto cualitativo, el rol de la teoría es significativamente menor, dependiendo también del diseño básico de la investigación; donde se destacan la teoría fundamentada, los diseños etnográficos, los diseños narrativos y los diseños de investigación-acción como los principales (Hernández, Fernández y Baptista, 2014, p. 470) observándose que en cada uno de ellos varía el rol de la teoría, pero en todo caso es significativamente menor que en la investigación cuantitativa.

Con respecto a la teoría fundamentada, por seleccionar algún diseño del enfoque cualitativo como ejemplo; sus autores diferencian entre los términos teoría sustantiva y formal, la primera se refiere a explicaciones de los informantes sobre su forma de percibir la realidad y la segunda se deriva del estudio por medio de un riguroso proceso de abstracción, (Strauss y Corbin, 2002, p. 26) donde se observa que efectivamente se usa, pero no como un marco interpretativo a partir de alguna conocida, sino todo el tiempo a partir de lo obtenido en la propia investigación, por lo que "es capaz de proporcionar teorías, conceptos, hipótesis, partiendo en forma directa de los datos y no de marcos teóricos ya establecidos con anticipación" (Giraldo, 2011, p. 80) es decir, en este caso es desarrollada por el investigador.

En otro orden de ideas, es conveniente revisar un poco como se define una teoría para tratar de comprender lo ocurrido, enmarcando, que se trata de un término polisémico pues existen múltiples definiciones de esta en el marco de las investigaciones, muchas de ellas coinciden en que se trata de una asociación sistemática de conceptos y proposiciones que sirven para resumir y relacionar ideas, contenidos y conocimiento sobre un tema determinado. Algunos autores incluso van más allá, como (Yurén, 2006, p. 33) que menciona "Una investigación llega a ser 'ciencia' cuando en ella se han construido teorías. Los datos, los problemas, las hipótesis y las leyes sueltas no constituyen una ciencia" colocándola 
entonces como elemento fundamental de la investigación, pero sin refutar por tanto la teoría fundamentada, en la que efectivamente ocurre la construcción mencionada.

Sin embargo, pensar en la construcción de una teoría sin el apoyo de alguna previa o incluso investigar sin usar ninguna, no parece del todo posible, pues, aunque sea de forma implícita, la manera de razonar del cerebro humano es comparativa, asociando con ideas preestablecidas. Un ejemplo de tal situación, sería observar una imagen de objetos completamente desconocidos, ubicados en un espacio también desconocido e intentar interpretar esa realidad. El cerebro entonces intenta dar sentido a la situación y la forma que tiene de hacerlo, es comparando con información conocida preguntándose "¿a qué se parece esto?", entonces compara con conceptos conocidos, estos últimos forman parte de datos obtenidos de experiencias y que fueron agrupados por el cerebro en ciertas categorías, la cuales pertenecen a una o varias teorías existentes, por ejemplo un objeto con forma cuadrada; siendo el cuadrado una figura geométrica, definida previamente en el pasado por Euclídes. Desde otro enfoque, esta idea también puede ser comprendida a partir del pensamiento popperiano:

Uno nunca lee o entiende un libro salvo con la ayuda de expectativas definidas que se encuentran en la propia mente. Esto puede considerarse como una de las consecuencias de mis tesis de que todo lo tratamos a la luz de una teoría preconcebida (Popper, 1975, p. 149)

Por todo lo anterior, a pesar de los esfuerzos de algunos diseños de investigación cualitativos por negar la importancia de las teorías, podría decirse que hacen uso de las mismas de forma implícita, tangencial o si se quiere utilizar un término que ya usan algunos de los diseños cualitativos, son un marco de referencia, término bastante apropiado a la realidad de lo que realmente ocurre en las investigaciones de ese enfoque.

Es importante destacar, que no se trata de darle a las teorías el grado de infalibles ni mucho menos; se trata de darle carácter científico a las investigaciones, enmarcándolas dentro de un conocimiento, el cual incluso puede ser refutado, por lo que estas tampoco son perfectas, así lo muestra (Lakatos, 1989, p.10) al mencionar "Realmente lo que caracteriza a la conducta científica es un cierto escepticismo incluso con relación a nuestras teorías más estimadas. La profesión de fe ciega en una teoría no es una virtud intelectual sino un crimen intelectual".

Por otra parte, cuando se revisa el enfoque cuantitativo, el acuerdo en el uso de las teorías es casi unánime, cualquiera que sea el diseño (experimental o no experimental) o el tipo de investigación (exploratoria, descriptiva, correlacional, explicativa, etc.), la teoría forma parte tanto de la metodología, como de todo el proceso asociado a los resultados y no solo en el campo de las ciencias básicas sino también las sociales.

La teoría es fundamental en la investigación social, aporta su origen (fuente de nuevos problemas e hipótesis) y su marco (proporciona el sistema conceptual) se aplica a la observación, clasificación y sistematización de los datos de la realidad (debe desembocar en teorías más perfectas). (Del Canto y Silva, 2013, p. 29)

Solo para tratar de ilustrar lo anterior, se hizo una revisión al azar de diez artículos científicos de ciencias sociales, para ver el rol que tenía la teoría en estos, su uso en la interpretación de los resultados de la investigación, encontrando como hallazgo interesante que el $20 \%$ de ellas (o sea 2 ) no solo no hacían uso de ninguna teoría en los resultados, sino a lo largo de toda la investigación, llegando incluso a no mencionar en todo el artículo la palabra teoría, teórico o teórica. 
De lo mencionado previamente, se tiene el artículo de Alzugaray, Mederoz y Sutz (2011) titulado "La investigación científica contribuyendo a la inclusión social" que tiene una extensión de 20 páginas y en las cuales no aparece mención alguna a las palabras asociadas a teoría, ni muestran indicios de uso de alguna y donde además curiosamente aparece en el título el término investigación científica, lo que al menos es contradictorio si se recuerda lo citado previamente por Yurén. Del mismo modo, se encontró el artículo de Castro y Olmos (2014) titulado "Características de las interacciones con la sociedad de los investigadores de humanidades y ciencias sociales a partir de estudios empíricos" con una extensión de 29 páginas en las que tampoco aparecen las palabras teoría, teórico o teórica y mucho menos se hace una interpretación de los resultados a partir de una teoría.

Cabe señalar, que el hecho de mencionar las investigaciones anteriores, más allá de cualquier crítica, tiene como propósito, mostrar casos donde la interpretación de los resultados es omitida de forma intencional, es decir los investigadores en estos casos no consideraron necesario el uso de teorías para obtener su producto final, enfocando su estudio en esos casos, estrictamente en elementos puramente empíricos, lo cual es una forma de trabajo válida en el enfoque cualitativo.

Sin embargo, no todos los casos de investigación cualitativa entran dentro de esta forma de trabajo, el diálogo entre la teoría y los resultados de la investigación, también ocurre en este enfoque y es ilustrado en el siguiente fragmento, refiriéndose a este como diálogo teórico empírico, dentro de un texto exclusivo de metodología cualitativa.

Si la investigación fue teórico-empírica, ello supone que hubo al inicio un trabajo de indagación teórica sobre el objeto de estudio (Estado del arte) y que durante el desarrollo de toda la investigación se estuvo leyendo y haciendo fichas bibliográficas extrayendo fragmentos de los textos teóricos involucrados, además de todo el trabajo empírico que supuso el levantamiento de campo. De modo que, al llegar a esta etapa se cuenta con la estructura empírica de los hallazgos en lógica de categorías, características, nodos, relaciones, relaciones de las relaciones o en cualquier otra forma que se haya inteligido hacer la síntesis empírica, pero también con una buena cantidad de fichas o fragmentos de texto teóricos que refieren de algún modo a aspectos de la estructura empírica obtenida. Es momento pues de desarrollar el diálogo teórico-empírico, es decir, de poner en relación los hallazgos empíricos estructurados -como quiera que ello se haya hecho-, y lo leído teóricamente. (Vargas, 2011, p. 70)

A partir de lo anterior, se destaca la importancia y posibilidad de interpretación de los resultados a partir de la teoría previa, incluso en la investigación cualitativa, por lo que, con independencia del enfoque, el uso de las mismas más que justificado sería necesario.

Corresponde revisar la otra vertiente, la omisión de la teoría en la discusión de resultados, cuando se trata de trabajos de grado y/o tesis. A partir de la función del presente investigador como jurado de tesis en pregrado y maestría, pero sobre todo en este último caso, se notó, cómo los investigadores autores de trabajos de grado para optar al título de magister en tecnología educativa, desarrollaban un marco teórico como una especie de accesorio en la investigación, se colocaba en el trabajo porque la estructura así lo exige, pero ni dentro de estos ni en las presentaciones públicas se observó, cual era la teoría principal y cuáles eran las de respaldo que basaba la investigación y lo más grave, se analizaban los resultados en ausencia, casi total de la teoría, aun tratándose de investigaciones de enfoque cuantitativo, lo que representa un problema metodológico, epistemológico, científico, entre otros. 
Es importante destacar, que el problema mencionado previamente, no es problema meramente local, ni una excepción a la regla, ciertamente ha sido identificado previamente desde hace algún tiempo como muestran (Bonilla y Rodríguez, 2005, p. 51) mencionando que "Aunque en sus propios fundamentos se confiere un papel central a la teoría en los métodos cuantitativos, en la realidad esta función se ha distorsionado" por esto se hace importante la revisión del mismo.

Lo que se observó, es que existe una tendencia a interpretar los resultados en forma de presentación de estadísticas, en su mayoría descriptiva y a la hora de interpretarlo, suelen responderse las hipótesis de inicio o interrogantes de la investigación de forma especulativa; es decir, opinando de forma personal sobre porque ocurren los hechos y a qué se deben las discrepancias con lo esperado, haciendo énfasis en las cifras obtenidas y justificando la realización de la investigación, pero con casi nula aparición de las teorías presentadas en el capítulo correspondiente a tal fin. Esto coincide con lo reportado por otros autores, como se muestra a continuación

Sin que haya ese posicionamiento se puede reducir el valor teórico de las conclusiones porque el investigador al hacerlas se ata demasiado mecánicamente a los resultados de los análisis de datos, sin hacer un esfuerzo de perspectiva más amplia, sin buscar lo que representan los resultados de la investigación en su conjunto. Posiblemente representan algo también para la teoría. (Dendaluze, 1997, p. 98)

Por lo tanto, el proceso de interpretación de resultados a la luz de la teoría, representa una pieza vital del trabajo del investigador y no puede ser ignorado, encubierto, puesto "de relleno" o como "accesorio", es precisamente en esta parte, donde se ve la coincidencia entre lo planteado inicialmente en las hipótesis o interrogantes y lo obtenido de forma empírica. En esta interpretación y precisamente en las hipótesis y/o interrogantes, es donde se ve la función del marco teórico, pues "conduce al establecimiento de las hipótesis, sugiere formas de análisis, o nuevas perspectivas a considerarse, y al mismo tiempo, ayuda a interpretar los resultados del estudio" (Reidl, 2012, p. 148).

Igualmente, es significativo resaltar que el proceso de interpretación de resultados depende de la cosmovisión del investigador, es una guía para el lector de la investigación, pero no debe ser tomado como una certeza sobre lo empírico. El lector, que incluso en algunas ocasiones suele tener más experiencia en el tema que el propio investigador, tiene a su mano los resultados obtenidos, de forma que pueda dar una interpretación distinta a estos, pudiendo refutar interpretaciones realizadas por el autor o incluso poniendo en duda la obtención de dichos resultados; de esto se trata la ciencia y es la forma como esta avanza.

Del mismo modo, en el apartado de interpretación, es donde el investigador puede exponer los elementos que permiten complementar la teoría existente a partir de los resultados obtenidos, llenar las brechas teóricas conocidas previamente 0 simplemente estar en discrepancia con elementos de la totalidad de las mismas, recordando que uno de los elementos principales de las teorías son las categorías, la cuales "son códigos interpretativos de la realidad que determinan lo que va a observar el investigador. Es la forma en que se aprehende la empírica" (Zambrano y Dueñas, 2016, p. 167) y precisamente estas categorías dependen de la cosmovisión del investigador, lo que le da mayor importancia al proceso de interpretación de resultados basado en las teorías.

Por todo esto, es importante como una posible solución al problema de la omisión de teorías en la interpretación de los resultados, reforzar el aspecto metodológico en la formación de los investigadores, con respecto a la presentación 
e interpretación de estos, el cual en líneas generales se trata en menor proporción que otros aspectos, esto ocurre en diversas áreas de estudio como se muestra a continuación para el caso del área de la salud

La categoría "resultado" no ha sido trabajada en los textos de metodología de la investigación, especialmente en aquellos que se usan con mayor frecuencia para las investigaciones en salud. Estos dedican la mayor atención a los elementos del diseño teórico-metodológico, al proceso, sus etapas y la elaboración del informe de la investigación; sin embargo, no refiere cómo elaborar la propuesta y presentar los aportes como resultados científicos (Travieso 2017, p. 612)

Esta situación, aunque se reporta para el caso de salud, también es observada en la mayor parte de los libros de texto de metodología que se usan en líneas generales, de lo que no se escapan los más usados en ciencias sociales y puede ser una de las múltiples causas de un problema que disminuye la calidad de las investigaciones y desmejora la forma como se hace ciencia, dentro de los espacios educativos en general.

\section{CONCLUSIÓN}

La interpretación de los resultados, es un apartado que aparece en casi todas las estructuras de las investigaciones propuestas en los diversos textos de metodología de la investigación, ya sean artículos científicos, trabajos de grado o tesis. Sin embargo, a veces no se realiza o cuando se hace, presenta una serie de problemas, siendo uno de los principales la omisión de las teorías. Según el estudio realizado, esta omisión proviene de dos vertientes, una intencionada relacionada a los diseños y enfoques de las investigaciones, principalmente el cualitativo y otra, la omisión debido a problemas metodológicos, observada en trabajos de grado a nivel de maestría.

Conviene destacar que, sin importar la vertiente estudiada, esta omisión representa un problema, pues es a partir de las teorías donde se pueden contrastar los resultados empíricos, no importa en líneas generales el enfoque utilizado. La interpretación de los resultados a través de estas, permite contribuir al conocimiento, enriqueciendo las mismas, logrando además llenar brechas teóricas existentes o refutar algunos aspectos de estas, llegando a ocurrir incluso que se pueda refutar una teoría en su totalidad. Todo esto, se ve reflejado en el desarrollo del conocimiento científico y en el fortalecimiento de la ciencia, en este caso particular de las ciencias sociales.

Finalmente, hay que señalar que una forma de revertir esta tendencia, sería fortaleciendo los contenidos y clases de metodología de la investigación impartidos en los espacios educativos, específicamente en el rol de la teoría dentro de la discusión de los resultados; además de ampliar y reconocer la importancia de esta parte de la investigación dentro de los textos de metodología, donde esto se observa en líneas generales como una debilidad y que se hace evidente, en la diferencia de espacio concedido a este aspecto con respecto a otros de igual o menor importancia.

\section{REFERENCIAS BIBLIOGRÁFICAS}

Alzugaray, S., Mederos, L., \& Sutz, J. (2011). La investigación científica contribuyendo a la inclusión social. Revista Iberoamericana de Ciencia, Tecnología y Sociedad, 6(17), 11-30. Obtenido de https://www.redalyc.org/pdf/924/92422634001.pdf 
Batthyány, K., \& Cabrera, M. (2011). Metodología de la investigación en ciencias sociales. Montevideo, Uruguay: Universidad de la República. Obtenido de http://cienciassociales.edu.uy/departamentodesociologia/wp-

content/uploads/sites/3/2013/

archivos/FCS_Batthianny_2011-07-27-imprimir.pdf

Bonilla, E., \& Rodríguez, P. (2013). Más allá del dilema de los métodos. Bogotá: Ediciones Uniandes. Obtenido de https://es.scribd.com/document/375854141/BONILLARODRIGUEZ-Mas-Alla-Del-Dilema-de-Los-Metodos-UPA

Cáceres, G. (2014). La importancia de publicar los resultados de Investigación. Revista Facultad de Ingeniería, 23(37), 7-8. Obtenido de https://www.redalyc.org/pdf/4139/413937008001. pdf

Castro, E., \& Olmos, J. (2014). Características de las interacciones con la sociedad de los investigadores de humanidades y ciencias sociales a partir de estudios empíricos. Revista CTS, 27(9), 113-141. Obtenido de https://www.redalyc.org/pdf/924/92431880007.pdf

Cazau, P. (2011). Evolución de las relaciones entre la epistemología y la metodología de la investigacióm. Paradigmas,, 3(1), 109-126. Obtenido de https://dialnet.unirioja.es/descarg a/articulo/3798214.pdf

Del Canto, E., \& Silva, A. (2013). Metodología cuantitativa: Abordaje desde la complementariedad en ciencias sociales. Revista CienciasSociales, 141(3), 25-34. Obtenido de https://www.redalyc.org/pdf/153/15329875002.pdf

Dendaluze, I. (1997). La validez teórica de las investigaciones empíricas en Ciencias Sociales. Revista Internacional de los Estudios Vascos, 42(1), 77-101. Obtenido de http://hedatuz.euskomedia.org/2251/1/42077101.pdf

Giraldo, M. (2011). Abordaje de la Investigación Cualitativa a través de la Teoría Fundamentada en los Datos. Ingeniería Industrial. Actualidad y Nuevas Tendencias, 2(6), 79-86. Obtenido de https://www.redalyc.org/pdf/2150/215021914006.pdf

Gomez, C. (2006). La investigación científica en preguntas y respuestas. Ambato, Ecuador: Corporación Uniandes. Obtenido de https://ebevidencia.com/wpcontent/uploads/2014/07/

La-investigacion-cientifica-ebevidencia.pdf

Hernández, R., Fernández, C., \& Baptista, P. (2014). Metodología de la investigación . México : McGraw Hill Education.

Lakatos, I. (1989). La metodología de los programas de investigación científica. Madrid: Alianza Editorial.

Popper, K. (1975). La ciencia normal y sus peligros. En I. Lakatos, \& A. Musgrave, La crítica y el desarrollo del conocimiento científico (págs. 149-158). Barcelona, España: Grijalbo. Obtenido http://datateca.unad.edu.co/contenidos/90018/TEORIA_DEL_CONOCIMIE

NTO/TEXTOS_UNIDAD_2/Popper_La_ciencia_normal_y_sus_peligros.pdf

Reidl, L. (2012). Marco conceptual en el proceso de investigación. Investigación en educación médica, 1(3), 146-151. Obtenido de http://www.scielo.org.mx/pdf/iem/v1n3/v1n3a7.pdf

Samaja, J. (2004). Epistemología y metodología. Buenos Aires: Editorial unversitaria de Buenos Aires.

Strauss, A., \& Corbin, J. (2002). Bases de la Investigación Cualitativa. Técnicas y Procedimientos para Desarrollar la Teoría Fundamentada. Bogotá, Colombia: Editorial Universidad de Antioquia.

Travieso, N. (2017). Los resultados científicos en las investigaciones biomédicas: un desafío pendiente. Medisan,
http://scielo.sld.cu/pdf/san/v21n5/san162 15.pdf

Vargas, X. (2011). ¿Cómo hacer investigación cualitativa? México: Etxeta. 
Yurén, M. (2006). Leyes, teorías y modelos. México: Trillas.

Zambrano, J., \& Dueñas, K. (2016). La articulación entre teoría, objetivos y metodología en la investigación social. Domino de las ciencias, 2(núm. esp.), 163-174. Obtenido de https://dialnet.unirioja.es/descarga/articulo/5802929.pdf 